\title{
Call for Proposals: Special Section of Archives of Sexual Behavior on "The Impact of Youth Violence on Sexual Health of Adolescents from National and International Perspectives"
}

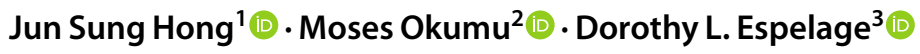

Published online: 13 October 2021

(C) The Author(s), under exclusive licence to Springer Science+Business Media, LLC, part of Springer Nature 2021

Addressing youth violence and promoting the sexual health of adolescents continue to be a public health priority across the globe. A significant body of empirical studies has demonstrated that many forms of youth violence are significant predictors of numerous adverse health and psychosocial outcomes, such as alcohol and drug use, delinquency, future violence, internalizing symptoms, and suicidal ideation and behavior.

Emerging literature has also documented that experiences of youth violence are correlated with negative sexual health outcomes. The association between youth violence and negative sexual health outcomes can be supported by several theoretical perspectives, including the self-medication hypothesis, social disorganization theory, feminist theory, and social control theory.

The Special Section aims to bring together scholars from around the globe to examine how youth violence might be related to the sexual health of adolescents. Youth violence consists of bullying (including cyberbullying), relational aggression, sibling violence, teen dating violence, gender-based violence, gang violence, non-consensual sexting, sexual violence/assault, poly-victimization (and poly-perpetration), and adolescent stalking behavior. Sexual health is conceptualized by risks, such as unwanted pregnancy, sexually-risky behaviors, multiple sex partners, sex under the influence of drugs, prostitution, and acquisition of sexually transmitted infections as well as protective

Jun Sung Hong

f14684@wayne.edu

Moses Okumu

okumu@illinois.edu

Dorothy L. Espelage

espelage@unc.edu

1 School of Social Work, Wayne State University, Detroit, MI 48202, USA

2 School of Social Work, University of Illinois at Urbana-Champaign, Urbana, IL 61801, USA

3 School of Education, University of North Carolina at Chapel Hill, Chapel Hill, NC 27599, USA elements, such as consent, condom efficacy, condom negotiation and use, sexual pleasure and well-being, sex education, and discussions with parents and teachers about sex. The Archives of Sexual Behavior invites proposals for this Special Section to highlight the impact of violence and victimization on the sexual health of children and adolescents.

The Special Section will include original, empirical research (quantitative, qualitative, and mixed methods), meta-analyses, and systematic reviews of the literature. We are especially interested in empirical studies that contain (1) a large adolescent sample (ages 10-18) and (2) an adolescent sample of minoritized identities (e.g., racial/ethnic minority, immigrant, refugees, LGBTQ+, etc.). Some topics of interest include (but are not limited to):

- Poly-victimization and sexually-risky behaviors of adolescents in urban neighborhoods

- Impact of sex education on sexually-risky behavior of victims of bullying

- Association between sibling violence and sex under the influence of drugs

- Technology-facilitated violence (e.g., cyberbullying) and how it is associated with a decision to engage in non-protected sex

- Sexual violence prevention associated with HIV-related risk practices among adolescents living in informal settlements

- Mechanisms underlying youth violence experiences and the sexual health of adolescents

- How does resilience shape the sexual health of adolescents who experience violence at first sex?

- How exposure to violence might be related to sex work

- Feminist perspectives on how youth violence is associated with the sexually-risky behavior of adolescent girls

Proposals should include type of article, title, names of all the authors, a brief abstract (250 words maximum), and 4-5 keywords. The abstract should include a statement of how the proposed study will contribute to the research literature. The 
proposed submission is required to have the following components: (1) cultural context of the country/region (e.g., a study conducted in Canada should have a Canadian context of youth violence and sexual health); (2) have one or more theoretical perspectives guiding the study; (3) describe specifically how the findings would build on extant literature and inform future research, practice, and policy. In addition, the following are required: (1) Times New Roman, font size of 12; (2) reference style of the American Psychological Association (7th ed.); (3) adherence to the journal guidelines for manuscript preparation (https://www.springer.com/journal/10508/submission-guide lines); (4) authors, not editors, bear the responsibility for ensuring accuracy in grammar, spelling, etc. If the author's native language is not English, use a professional company if necessary; (5) authors are solely responsible for the accuracy of the study data and research ethics. The research needs to be completed by the manuscript deadline.

For any questions about the special section or suitability of the topic, please contact Drs. Jun Sung Hong (fl4684@ wayne.edu), Moses Okumu (okumu@illinois.edu), and Dorothy L. Espelage (espelage@unc.edu). Please submit proposals to Dr. Jun Sung Hong (fl4684@wayne.edu) with the subject heading "Archives of Sexual Behavior Special Section Proposal." The deadline for the proposal is December 31, 2021. Decisions about the proposal will be sent out by February 15, 2022.

The full manuscript of approved proposals can be submitted by or before the deadline. Manuscripts need to be submitted to the journal's submission portal (not to the editors). Create an account and click on "Submit New Manuscript" and select article type "Youth Violence and Sexual Health." Please read the instructions for authors very carefully before submitting the manuscript. In the cover letter, please address it to "Drs. Hong, Okumu, and Espelage" and include the names and email addresses of five independent reviewers who might be suitable to review the manuscript. The deadline for manuscript submission is April 15,2022

The accepted manuscript will be published on the Archives of Sexual Behavior website on an ongoing basis. The Special Section is expected to be published in the December 2022 print issue.

Publisher's Note Springer Nature remains neutral with regard to jurisdictional claims in published maps and institutional affiliations. 